\title{
LA-UR $96-3168$
}

TITLE: SHOCK COMPRESSION SCIENCE:

Dynamic Material Properties and Computation

RECEVED

OCT 11 १ิง

OSTI

AUTHOR(S): James N. Johnson, T-1

SUвMITtED to: For Informal Distribution

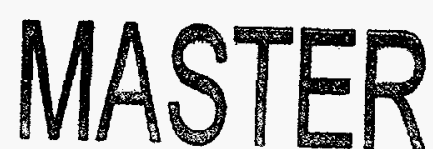

DISTRIBUTION OF THIS DOCUMENT IS UNLHMTEI PS

By acceptance of this article, the publisher recognizes that the U.S. Government retains a nonexclusing, royalty-free license to publish or reproduce the published form of this contribution, or to allow others to do so, for U.S. Government purposes.

The Los Alamos National Laboratory requests that the publisher identify this article as work performed under the auspices of the U.S. Department of Energy 


\section{DISCLAIMER}

Portions of this document may be illegible in electronic image products. Images are produced from the best available original document. 


\section{Shock Compression Science: Dynamic Material Properties and Computation}

James N. Johnson

Los Alamos National Laboratory

\section{Introduction}

Constitutive models used in computational prediction of high-rate deformation related to SBSS are determined by validation experiments that can be interpreted with some degree of uniqueness and without ambiguity. Here we include not only material strength and fracture, but also equation-of-state information, and, in the case of reactive solids, initiation concepts related to material strength and fracture.

By equation of state we mean material information derived from equilibrium thermodynamics. For fluids this is normally the $\mathrm{p}, \mathrm{V}, \mathrm{E}, \mathrm{T}$ information necessary for high-pressure shock-wave applications. For elastic solids it can also be second- and third-order adiabatic elastic moduli for crystals of arbitrary symmetry. Material strength and fracture are generally related to the defect state of the material, and are thus distinct from equilibrium thermodynamics. Likewise, initiation of solid explosives is related to hot spots arising from defects such as porosity and cracking. Finally, by the term "validation" we mean the repeated comparison of a computational model with a set of interpretable experiments, each exercising a particular aspect of the model under wellcontrolled loading conditions.

To be explicitly clear on the thrust of this section, emphasis is on the experimentation necessary to define and elucidate models. Here the role of computation is in the analysis of experiments. Of course, once the validity of a model is established with some confidence, large-scale computation can be used as a predictive tool.

\section{General}

An important aspect to be addressed in this document is the trade-off, or balance, between constitutive information (as determined by innovative and probing experimentation combined with the corresponding analysis) and specifically computational questions such as code architecture, variable storage, and computational 
speed. This distinction is important because it appears that we may have become somewhat polarized into two camps. It is the view of this committee that, in assessing the balance between these two distinct and legitimate disciplines, computational progress has run far ahead of our ability to characterize material behavior. This seems to have happened by chance rather than design. Natural scientists interested in material properties have intuitively suspected that constitutive behavior is at least as important as the detailed geometry that can now be included in continuum code calculations. However, the natural scientists may have been derelict in making compelling, objective arguments to the computational scientists for the importance of improved material models. Likewise, computational scientists have found in the past that elaborate material models often slow down calculations excessively without giving improved predictions.

With regard to computational speed we recognize that much of the resources and improvements in this area have recently gone into developing the ability to handle threedimensional geometry. When it may have been possible to bring material modeling up to a level commensurate with geometry and zoning improvements in one- and twodimensional codes, there was always the lure of the third dimension. Well, we are now there. There are no more spatial dimensions to include. The next horizon is material behavior. It is incumbent upon shock compression and detonation scientists to provide compelling evidence for this view.

The most serious negative influence on successful computational modeling is unanticipated material behavior. Such unanticipated effects are uncovered only by inventive and adventurous experimentation. Here we want to give two examples where improved material modeling pays its way with respect to the loss of computational speed. These are examples of past successes related to nuclear weapons design and hence are applicable to the SBSS thrust; they are presented here only as aids in visualizing the benefits to be realized by an invigorated research program in Shock Compression and Detonation Science.

Shock Desensitization of Solid Explosives.

The definition of shock desensitization is the following: preshock insufficient to cause full detonation tends to desensitize the material to the next shock. This is simply because low-amplitude shocks do not produce hot spots, but rather what might be called "lukewarm spots." Because of Arrhenius kinetics, lukewarm spots take a long time to 